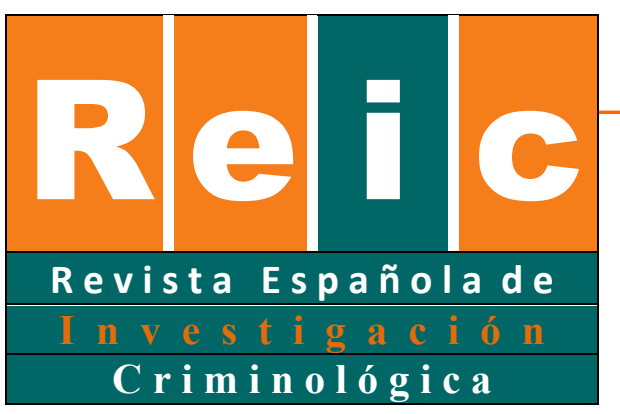

Meléndez

\title{
El papel de la mediación penal en el proceso de cambio del infractor
}

\section{The role of victim-offender mediation in the offender's process of change}

\author{
Anna Meléndez 1 \\ Universitat Autònoma de Barcelona
}

\section{RESUMEN}

El objetivo principal de este artículo es conocer la capacidad de la justicia restaurativa de incidir en el desistimiento del comportamiento antisocial o delictivo, con el fin de identificar el rol de la mediación en los potenciales procesos de cambio (Prochaska \& DiClemente, 1983 ; 1984) de los infractores. Los resultados muestran que es posible identificar infractores en todos los estadios del cambio. Entre los diversos factores relacionados con la motivación y con el proceso de cambio, la mayoría de los infractores identifican la mediación como un punto de inflexión o elemento significativo en ese camino hacia la vida prosocial. Los resultados muestran la complejidad del proceso de cambio y la dificultad que los infractores tienen a la hora de conseguir, pero sobre todo de mantener, este cambio.

Palabras clave: justicia restaurativa, mediación penal, desistimiento, proceso de cambio

\begin{abstract}
The main objective of this article is to examine the capacity of restorative justice to have an influence on desistance from antisocial or criminal behaviour, in order to identify the role of mediation in the process of change (Prochaska \& DiClemente, 1983; 1984) of offenders. Results show that it is possible to identify offenders in all of the stages of change. Among the factors linked to motivation and the process of change, the vast majority of the offenders identify mediation as a turning point or a significant element on the way to a pro-social life.
\end{abstract}

${ }^{1}$ La correspondencia debe dirigirse a: anna.melendez@uab.cat

Revista Española de Investigación Criminológica

Artículo 11, Número 16 (2018)

ttps://doi.org/10.46381/reic.v16i0.189

Www.criminologia.net

ISSN: 1696-9219 
Results show the complexity of the process of change and the difficulties offenders have to face in order to achieve it, and specially the challenge of maintaining this change.

Keywords: restorative justice, victim-offender mediation, desistance, process of change.

\section{Introducción}

Este trabajo tiene como objetivo explorar la vinculación del modelo restaurador con la perspectiva teórica del desistimiento. Esta aproximación se justifica en la necesidad de conocer la capacidad de la justicia restaurativa (en adelante JR) de incidir en el cese de la actividad antisocial, explorando y analizando los posibles mecanismos explicativos existentes entre la participación en procesos restaurativos y el proceso de abandono de la delincuencia, en particular, de los infractores participantes en procesos restaurativos.

El modelo restaurativo es una forma de responder al comportamiento delictivo a través de un balance entre las necesidades de las partes y la reparación de la comunidad, las víctimas y la de los infractores (Braithwaite, 2002a, 2002b; Christie, 1977; Dandurand \& Griffiths, 2006). Se fundamenta en la interacción activa y conjunta para la resolución de los conflictos derivados del delito, con la ayuda de un tercero facilitador (Marshall, 1999). A pesar de que hay diversas perspectivas teóricas (Braithwaite \& Strang, 2000; Dignan, 2005; Hoyle \& Fonseca, 2016; Johnstone \& Van Ness, 2007a, 2007b; Walgrave, 2007), todas parten de la existencia de un conflicto.

El desistimiento es un proceso gradual de abandono de la actividad delictiva que finalizará cuando la persona deje de delinquir e irá acompañado normalmente de una narrativa de cambio (Bottoms, Shapland, Costello, Holmes, \& Muir, 2004; Robinson \& Shapland, 2008). A pesar de existir diversas definiciones, Mulvey et al. (2004) consideran adecuado el uso de definiciones integradoras de desistimiento. En este sentido, consideran que para medir el desistimiento se deberían demostrar tres cosas: la disminución del nivel (gravedad) de la conducta antisocial en un periodo de tiempo razonable; el declive de la frecuencia de la actividad delictiva; y la tercera, tiene que ver con la necesidad de poder

Revista Española de Investigación Criminológica

Artículo 11, Número 16 (2018)

ttps://doi.org/10.46381/reic.v16i0.189

www.criminologia.net

ISSN: 1696-9219 
distinguir entre la reducción de un delito en concreto o un declive general de la actividad antisocial.

En particular, en este artículo se parte de un marco teórico que avala la integración de la justicia restaurativa y el desistimiento (Maruna, 2014; Shapland, 2013) tomando como elemento teórico explicativo la teoría del cambio de Prochaska y DiClemente (1983; 1984).

En cuanto al vínculo entre ambos modelos teóricos, Maruna (2014) ha destacado que es posible conectar ideas e integrar conceptos. En primer lugar, Maruna (2014) considera que el desistimiento puede aprender del modelo restaurativo la habilidad de utilizar el concepto de delincuencia desde una perspectiva humanizadora, así como la idea de entender el delito como un conflicto entre individuos (Zehr, 1990). En segundo lugar, el autor cree que la JR puede ayudar a comprender emociones como la vergüenza, la culpa o el orgullo, tal y como han destacado otros autores (Harris, Walgrave, \& Braithwaite, 2004; Harris, 2006; Rossner, 2011; Scheff \& Retzinger, 1991; Van Stokkom, 2002). Estas emociones son importantes para comprender el comportamiento delictivo y además permiten cuestionar el rol predominante que se tiende a dar del delincuente como un ser esencialmente racional. La tercera idea a la que Maruna (2014) hace referencia es a las micro-dinámicas que se producen en los encuentros restaurativos, siendo en el nivel micro-social donde es posible reconocer el rol que los rituales y las emociones tienen. Es en este contexto donde es posible que las personas puedan asumir responsabilidades y arrepentirse de lo ocurrido con la ayuda de los profesionales (Maruna, 2001), pero sobre todo es el lugar idóneo para eliminar etiquetas estigmatizantes y hacer propuestas de mejora para el futuro. Este sentido de control de la propia vida y tener un plan de futuro es una forma de responsabilizarse y de iniciar un proceso de cambio. Las prácticas restaurativas son procesos sociales que facilitan la puesta en común de las vivencias de los participantes, que pueden expresarse con sinceridad. En particular para el infractor el hecho de escuchar a la víctima le permite reflexionar y trabajar en las razones para dejar de delinquir (Strelan, Di Fiore, \& Van Prooijent, 2017). En este sentido estos encuentros restauradores pueden facilitar narrativas de cambio, que ayuden a construir la identidad individual (Giordano, Cernkovich, \& Rudolph, 2002; Maruna, 2001). Además,

Revista Española de Investigación Criminológica

Artículo 11, Número 16 (2018)

ttps://doi.org/10.46381/reic.v16i0.189

www.criminologia.net

ISSN: 1696-9219 
generan la necesidad de iniciar el proceso de cambio, partiendo de la restauración o reparación del daño causado.

Por lo que se refiere a lo que la JR podría aprender del desistimiento, como primera idea, la teoría del desistimiento tiene una visión amplia y a largo plazo, en el sentido que se interesa por el futuro (Maruna, 2001). En segundo lugar, el desistimiento reconoce y destaca la importancia de los agentes externos y contextuales, elementos esenciales en el proceso individual de cambio del infractor. Sin embargo, la JR se centra más en el individuo que en su contexto -en función del rol que tenga la comunidad en cada proceso de JR-. Por último, el modelo teórico del desistimiento integra la emoción de la vergüenza en el proceso de reconstrucción de la identidad del infractor. Estos tres aspectos no suelen estar presente en las prácticas restaurativas o en su caso no las orientan, pero podrían integrarse para trabajar en clave restaurativa y desistente.

Shapland (2013), por su parte, vincula los dos modelos teóricos desde la perspectiva de los elementos que ambos modelos comparten (Bottoms et al., 2004; Bottoms \& Shapland, 2011; Shapland et al., 2007, 2011). La primera idea tiene que ver con la participación. Los participantes en los procesos restaurativos son potenciales desistentes. El hecho de que estos infractores accedan voluntariamente a participar asegura que están preparados mentalmente para encontrarse con las víctimas y hablar del delito. El segundo aspecto que Shapland (2013) identifica tiene que ver con el proceso restaurativo como un elemento que facilita y garantiza la comunicación. Es el lugar para expresarse, para pedir perdón, responder preguntas o mostrar altruismo hacia la víctima. Puede ser también el momento oportuno para poder separar la persona del delito cometido e intentar orientar la comunicación hacia el planteamiento de un futuro pro-social. Para el desistimiento la comunicación puede servirles a los infractores para expresar la voluntad de cambio, plantear las cosas que deberían cambiar y visualizar el tipo de persona que les gustaría llegar a ser. En tercer lugar, la autora destaca que los encuentros restaurativos ofrecen la oportunidad de cerrar una etapa, la delictiva, mediante la responsabilización y el perdón; para abrir una nueva, de carácter pro-social. En particular Shapland (2013) pone de relieve que el hecho de pedir disculpas significa admitir el error que supone haber cometido un delito y puede contribuir a adquirir compromisos para

\section{Revista Española de Investigación Criminológica}

Artículo 11, Número 16 (2018)

ttps://doi.org/10.46381/reic.v16i0.189

www.criminologia.net

ISSN: 1696-9219 
cambiar aspectos de la vida, como símbolo de reparación. Esta oportunidad en el lenguaje del desistimiento puede interpretarse como el momento adecuado para compartir con otras personas la decisión de desistir, significando para el infractor el fin de un tipo de vida que quiere cambiar. Además, Shapland (2013) considera de gran importancia los acuerdos a los que las partes pueden llegar al final de un proceso restaurador. El contenido de los acuerdos puede implicar compromisos de futuro, tales como la voluntad de dejar de delinquir acompañada de acciones que le permitan conseguir tal objetivo. Un encuentro restaurativo puede ser el contexto ideal para reafirmarse en la voluntad de desistir, que en el lenguaje del desistimiento se traduciría en un contexto que refuerza positivamente la posibilidad de cambiar hacia una vida pro-social. Por último, Shapland (2013) menciona la importancia del apoyo que tanto profesionales como personas cercanas ofrecen para contribuir al aumento del capital social del infractor, perspectiva compartida por ambos modelos teóricos.

La teoría que, a mi juicio, permite integrar estos dos paradigmas es la del cambio individual propuesta por Prochaska y DiClemente (1983, 1984). El cambio individual, entendido en parte como el abandono del comportamiento antisocial, es uno de los objetivos de los programas restaurativos. De acuerdo con Presser y Van Voorhis (2002), la restauración, entendida como un paso hacia el cambio, es indicativa de la garantía de la paz social, pero también individual. En este sentido, la restauración es capaz de promover el crecimiento personal y el cambio (Daly, 2003; Presser \& Van Voorhis, 2002; Roberts, 2010).

Prochaska y DiClemente $(1983,1984)$ han formulado un modelo teórico general para explicar la complejidad de cambiar el comportamiento. De acuerdo con este modelo teórico, el cambio de comportamiento es parte de un proceso que cuenta con diversos estadios. El primer estadio de cambio es la pre-contemplación y es un momento en que el individuo aún no ha considerado la opción de cambiar. En el segundo estadio, llamado contemplación, después de un proceso de reflexión la persona percibe que tiene un problema y empieza a considerar la posibilidad de cambiar. El tercer estadio supone un paso hacia adelante en este proceso, la preparación. Es el momento de la toma de decisión, una vez la persona está preparada para cambiar porque ya ha tomado la decisión de hacerlo, sin embargo aún no ha realizado ninguna acción. Para hacerlo, la persona necesita pasar al siguiente estadio, que

Revista Española de Investigación Criminológica

Artículo 11, Número 16 (2018)

ttps://doi.org/10.46381/reic.v16i0.189

www.criminologia.net

ISSN: 1696-9219 
consiste en la acción del cambio e incluye la planificación a corto plazo de aquellas acciones que constituirán propiamente el cambio. Este momento conlleva efectivamente la realización de alguna actividad o acción de cambio en particular. Por último, las autoras prevén el estadio del mantenimiento del cambio, que responde a una de las etapas de más reto y dificultad que las personas que ese encuentra en este proceso tienen que hacer frente. Los períodos de recaídas también se contemplan en el modelo teórico y encajan con la idea de concebir el cambio como un proceso.

El movimiento entre los distintos estadios puede verse influenciado por diversas variables (Prochaska \& DiClemente, 1983,1984). En primer lugar, las autoras se refieren al obstáculo que pueden plantear los pros y contras que las personas motivadas a cambiar pueden tener, como por ejemplo el dilema de abandonar o no el comportamiento antisocial. Un segundo aspecto que puede incidir en el proceso tiene que ver con la auto-eficacia, la confianza en la propia capacidad de llevar a cabo las acciones que conducen al cambio. Un tercer elemento que puede anteponerse en este proceso es la tentación de volver a retomar el comportamiento anti-normativo para hacer frente a las adversidades, lo que en ocasiones se traducirá en recaídas. Finalmente otro aspecto que va a tener mucha importancia en el éxito o fracaso del proceso serán las acciones empleadas -de índole diversa en función de cada persona- para conseguir este reto.

Idealmente, el proceso restaurativo permite a sus participantes explorar sobre sus sentimientos y emociones, así como aumentar la comprensión acerca de lo sucedido en el contexto de la infracción penal. En términos generales, esta teoría contribuirá a entender la influencia de los procesos restaurativos en el desistimiento de los infractores que forman parte de este estudio, así como a identificar el vínculo entre los dos paradigmas de acuerdo con los dos autores que así lo han manifestado (Shapland, 2013; Maruna, 2014). En particular, la aportación de la teoría del cambio individual es entender cuál es la situación inicial en el proceso del cambio que tiene cada uno de los participantes, y cuál es la capacidad que muestran en llevarlo a cabo satisfactoriamente.

Revista Española de Investigación Criminológica

Artículo 11, Número 16 (2018)

ttps://doi.org/10.46381/reic.v16i0.189

www.criminologia.net

ISSN: 1696-9219 


\section{Método}

\subsection{Introducción}

El objetivo principal de esta investigación consiste comprobar si la JR, en particular el proceso de mediación puede influir en el proceso de desistimiento.

El diseño de la investigación de tesis de la que parte el contenido de este artículo se inspira en uno de los modelos de evaluación de los programas restaurativos más utilizados, aunque no el único (Daly, 2003; Shapland et al, 2007; Varona, 2008). El modelo se desarrolla en tres fases: la primera se realiza en un momento previo al inicio del proceso (pre-proceso); la segunda implica el seguimiento; y la tercera requiere de un análisis posterior (postproceso). Este modelo se complementa con la inclusión de un modelo de evaluación en clave de desistimiento, adaptado a las particularidades del contexto. El estudio del desistimiento se realiza de manera prospectiva ${ }^{2}$ en la fase post-proceso, tal y como han hecho ya otros autores (Bottoms \& Shapland, 2010; Cid \& Martí, 2012), que tiene lugar en la finalización del proceso.

Si bien este artículo se centra en una parte muy concreta de toda la investigación, es necesario ubicarla para comprender mejor su contenido.

El trabajo de campo se divide en dos fases con cuatro momentos distintos. La primera fase se comprende de tres momentos (pre-proceso, proceso y post-proceso). La segunda fase consiste en una entrevista narrativa a los infractores de los procesos observados pasados seis meses de la mediación. Esta es la técnica utilizada para obtener los resultados en relación con el proceso de cambio de los infractores participantes en el estudio, que es el objeto de este artículo. Por este motivo, esa es la única técnica que se desarrolla con detalle.

\footnotetext{
${ }^{2}$ Un análisis prospectivo pretende realizar el seguimiento después del cumplimiento de las penas, en este caso puede equipararse a la finalización del proceso y del cumplimiento de los acuerdos.

Revista Española de Investigación Criminológica

Artículo 11, Número 16 (2018)

ttps://doi.org/10.46381/reic.v16i0.189

Www.criminologia.net

ISSN: 1696-9219
} 


\subsection{Muestra}

La condición inicial de inclusión en la investigación es ser mayor de edad, parte denunciada (infractor) y participar en un proceso de mediación, en el marco del programa de mediaciónreparación penal del Departamento de Justicia de la Generalitat de Catalunya, entre los meses de junio 2012 y julio de 2013. La muestra es de carácter intencional, basada en la selección de aquellos casos que hayan finalizado el programa con mediación directa o indirecta. De los 40 casos, solamente 12 llegan a ser entrevistados en los seis meses posteriores.

El objetivo es garantizar la diversidad de narrativas de desistimiento y persistencia, buscando así la saturación del discurso. Además, el proceso debe tener lugar en la provincia de Barcelona. Los participantes son hombres y mujeres entre 18 y 70 años, todos cometieron faltas -ahora delitos leves- de diversa índole (lesiones, amenazas, injurias, hurtos, estafas, desorden público) y las víctimas fueron tanto personas físicas como jurídicas.

\subsection{Técnica de recogida y análisis de datos: la entrevista}

La entrevista es de tipo narrativo y se realizó a 12 de los participantes en alguno de los 40 procesos de mediación observados y que habían respondido a los cuestionarios pre y post proceso. Se realizó un estudio de caso por tipologías. Todas las entrevistas tuvieron una duración de entre 40 y 60 minutos y fueron grabadas y transcritas. Fueron analizadas de acuerdo con categorías vinculadas a la hipótesis de partida.

El contenido de la entrevista fue diseñado para analizar cuatro elementos que la literatura del desistimiento ha destacado como relevantes: el pasado, con el comienzo de la conducta antisocial; el impacto del proceso de mediación - intervención- ; el proceso actual de transición, y finalmente las perspectivas de futuro del infractor.

Revista Española de Investigación Criminológica

Artículo 11, Número 16 (2018)

ttps://doi.org/10.46381/reic.v16i0.189

www.criminologia.net

ISSN: 1696-9219 


\section{Resultados}

\subsection{Proceso de mediación y desistimiento}

Los resultados obtenidos fruto del análisis de la primera parte de la investigación muestran la influencia del proceso de mediación en 6 variables de impacto y abordadas durante el proceso de mediación - responsabilidad, aceptación del daño, aceptación de la existencia de una víctima, reflexión, arrepentimiento y vergüenza-, de las que se esperaba obtener un cambio positivo a lo largo del proceso de mediación y una vez finalizado el mismo. Sin embargo, los resultados son diversos y en los 12 casos estudiados es posible identificar infractores en todos los grupos (desistentes y persistentes).

En cuanto a los resultados de desistimiento- entendido como no haber delinquido en los seis meses posteriores al proceso de mediación-, obtenidos de la entrevista realizada en segunda fase, hay infractores desistentes y persistentes. Algunos encajan con la idea de haber tenido un proceso de mediación positivo, mientras que para otros la mediación no es relevante en términos generales para hablar de desistimiento.

Existe un grupo de 4 persistentes, de los 12 infractores entrevistados. Tres de ellos han reincidido en el contexto del mismo conflicto que los llevó a la mediación, mientras que uno de ellos cometió una infracción contra otra víctima y además de carácter más grave. Los infractores persistentes pueden a su vez clasificarse en dos grupos. El primero incluye a dos infractoras que durante todo el proceso mantuvieron resultados negativos y que acabaron el proceso sin conseguir ningún cambio positivo, lo que tiene como consecuencia esperada la reincidencia. Los resultados obtenidos en este sentido eran los esperados en estas circunstancias. El segundo grupo se encuentran dos infractores que obtuvieron resultados positivos durante el proceso de mediación, pero que no obstante reincidieron. Si bien lo esperado es que no reincidieran, no pudieron evitar volver a delinquir.

En cuanto al grupo de los 8 desistentes incluye infractores que cambiaron a positivo, que lo hicieron a negativo o que no cambiaron. Entre los que cambiaron a positivo es el resultado óptimo y esperado. También en este grupo de desistentes se encuentran aquellos que no cambiaron, pero se mantuvieron positivos durante todo el proceso, para quienes el

\section{Revista Española de Investigación Criminológica}

Artículo 11, Número 16 (2018)

ttps://doi.org/10.46381/reic.v16i0.189

www.criminologia.net

ISSN: 1696-9219 
proceso les ayudó a trabajar la responsabilización responsabilidad y la auto-eficacia para iniciar el proceso de cambio.

Por último, es posible identificar un tercer grupo que a pesar de tener un proceso negativo - y que lo esperado hubiera sido la reincidencia- desistieron de su actividad anti normativa. Todos estos tienen en común que hicieron mediación indirecta y le dieron un uso instrumental, querían resolver el problema sin necesitar profundizar en la forma de hacerlo. Todos ellos tenían un perfil pro-social, lo que les ayudó a mantenerse al margen del comportamiento anti-normativos, que lo definían más como un error que como un estilo de vida.

\subsection{Proceso de cambio}

Una vez presentado como se distribuyen los 12 infractores, que fueron entrevistados seis meses después del proceso de mediación en relación a los cambios inmediatos después del proceso; y cómo esos cambios se plasmaron en su implicación o desistimiento, ahora se pretende analizar con detalle cómo es dicho proceso para cada uno de los infractores, tomando como punto de partida los estadios del cambio propuestos por Prochaska \& DiClemente $(1983,1984)$

Tabla 1. Evolución estadios del cambio previo, durante y seis meses después de la mediación

\begin{tabular}{|c|c|c|c|c|}
\hline Infractores & Estado cambio inicial & $\begin{array}{c}\text { Proceso justicia } \\
\text { restaurativa }\end{array}$ & Estado del cambio final & $\begin{array}{c}\text { Desistimiento } \\
\text { persistencia }\end{array}$ \\
\hline Rosa & Pre-contemplación & Sin Impacto & Pre-contemplación & Persistencia \\
\hline María & Pre-contemplación & Sin Impacto & Pre-contemplación & Persistencia \\
\hline Fátima & Pre-contemplación & $\begin{array}{l}\text { Impacto positivo- } \\
\text { Aprendizaje }\end{array}$ & Pre-contemplación & Desistimiento \\
\hline Marc & Pre-contemplación & Sin Impacto & Pre-contemplación & Desistimiento \\
\hline Jordi & Pre-contemplación & Sin Impacto & Pre-contemplación & Desistimiento \\
\hline Marcel & Pre-contemplación & Sin Impacto & Pre-contemplación & Desistimiento \\
\hline Juan & Contemplación & $\begin{array}{l}\text { Imapcto positivo- } \\
\text { Preparación- } \\
\text { Decisión }\end{array}$ & Recaída & Persistencia \\
\hline
\end{tabular}


Meléndez

\begin{tabular}{|l|l|l|l|l|}
\hline Mohamed & Contemplación & $\begin{array}{l}\text { Imapcto positivo- } \\
\text { Preparación- } \\
\text { Decisión }\end{array}$ & Recaída & Persistencia \\
\hline Carlos & $\begin{array}{l}\text { Preparación cambio- } \\
\text { Decisión }\end{array}$ & $\begin{array}{l}\text { Impacto positivo- } \\
\text { preparación- } \\
\text { planificación }\end{array}$ & Acciones y mantenimiento cambio & Desistimiento \\
\hline Pau & $\begin{array}{l}\text { Preparación cambio- } \\
\text { Decisión }\end{array}$ & $\begin{array}{l}\text { Impacto positivo- } \\
\text { preparación- } \\
\text { planificación }\end{array}$ & Acciones- recaída-nuevas acciones & Desistimiento* \\
\hline Jose & $\begin{array}{l}\text { Preparación cambio- } \\
\text { Decisión }\end{array}$ & $\begin{array}{l}\text { Impacto positivo- } \\
\text { preparación- } \\
\text { planificación }\end{array}$ & Acciones y mantenimiento cambio & Desistimiento \\
\hline Jonathan & $\begin{array}{l}\text { Preparación cambio- } \\
\text { Decisión }\end{array}$ & $\begin{array}{l}\text { preparación- } \\
\text { planificación }\end{array}$ & Acciones y mantenimiento cambio & Desistimiento \\
\hline
\end{tabular}

*Desistimiento con recaídas en el proceso

\section{Pre-contemplación ${ }^{3}$}

En este estadio, la persona no piensa en la necesidad de cambiar. Entre los 12 casos, 6 se clasifican en esta categoría. A su vez es posible distinguir infractores con un pasado conflictivo y otros con uno pro-social.

\section{Pasado conflictivo y persistentes}

En este grupo se encuentran Rosa y María, infractoras que no obtuvieron ningún efecto positivo de la mediación. Para ellas la mediación no significó ni un punto de inflexión ni tampoco un ímpetu para el cambio. Tampoco es posible detectar narrativas de cambio en su discurso a lo largo del proceso o en la entrevista final. Ambas coinciden en no haber aprendido nada que aplicar a sus vidas del proceso. El hecho de situarse en un estadio de precontemplación hace que fueran poco sensibles a la mediación y, por tanto, es comprensible que esta no tuviera ninguna influencia en su actividad antisocial posterior. Claramente no se plantean la necesidad de cambiar y se encuentran estancadas en este momento, pues no son

\footnotetext{
${ }^{3}$ Las citas que ejemplifican la exposición de los resultados han sido extraídas de las 12 entrevistas realizadas seis meses después de la mediación. Por cuestiones de espacio solamente se acompañarán de citas algunos de los resultados, el resto se comentarán de acuerdo con las entrevistas transcritas y analizadas. Las intervenciones de la entrevistadora se destacan en negrita.
}

Revista Española de Investigación Criminológica

Artículo 11, Número 16 (2018)

ttps://doi.org/10.46381/reic.v16i0.189

Www.criminologia.net

ISSN: 1696-9219 
capaces de replantear sus posturas acerca del conflicto que tuvieron y que siguen teniendo con las mismas personas. Estos casos representan en cierta medida el fracaso de la mediación.

\section{Pasado pro-social y desistentes}

En este grupo los infractores tampoco se habían planteado cambiar, pero en este caso los miembros de este grupo coinciden con ser escépticos acerca de esta necesidad, en el sentido que son personas con una vida totalmente pro- social, que dicen cometer errores de vez en cuando. Todos, sin embargo, respondieron en el auto-informe haber cometido infracciones penales en el pasado. Respecto al proceso de mediación, coinciden en que no es el lugar adecuado para plantearse cambios en sus vidas, sino un espacio para lidiar y resolver un problema concreto sin la necesidad de ir más allá. Marc y Jordi querían únicamente pasar página y creyeron que la mediación era una forma rápida y fácil para conseguirlo. Fátima, a diferencia de Marc y Jordi, durante la entrevista muestra un cambio de perspectiva en relación con el conflicto y a la mediación, que considera que sí obtuvo un aprendizaje: sobre todo destaca la capacidad reflexiva como un aspecto que mejoró. En definitiva, su perfil pro-social puede explicar por qué no reincidieron, pero no su participación en el proceso de mediación, que fue de carácter instrumental.

\section{Contemplación}

El segundo estadio de cambio es la contemplación. En este momento la persona advierte que tiene un problema y empieza a pensar en la necesidad de cambiar. Este es el estadio al que algunos de los infractores fueron capaces de llegar durante el proceso restaurativo. Varios de los infractores de este grupo consideran que el mediador tuvo un rol muy importante, por ser respetuoso y sobre todo porque los humanizó. Los infractores se sintieron apoyados por este profesional, en particular mencionan que les ayudó a pensar en la necesidad de cambiar. En algunos casos, la mediación fue percibida por los infractores como una segunda oportunidad, en la medida que se generó una atmosfera constructiva en la que los participantes no se sintieron etiquetados como delincuentes. 
Para este grupo la mediación representó un elemento de cambio, un lugar dónde los infractores se dieron cuenta de las consecuencias de su comportamiento, que todavía podían reparar. En esta situación se encontraba tanto Juan como Mohamed. Ambos, además de plantearse la necesidad de cambiar, también terminaron tomando la decisión de hacerlo durante el proceso de mediación, sin embargo, ninguno de los dos lo consiguió, volvieron a delinquir.

(...) desde que acabó la mediación, ¿qué cosas te han pasado importantes que podrías destacar?

Me han pasao muchas cosas! Qué quieres que te diga? hace dos meses o tres $m$ 'han pillao, dos veces detenido, muchas cosas...

¿Y qué te ha pasado, qué has hecho para que te detuvieran?

Muchos problemas...es que no lo quiero decir...es que todavía tengo juicios pendientes... Sabes, por robos! Me han venido a buscar a casa por un robo en un coche. Y me han llevao casi tres dias al calabozo y luego a juicio (...)

(Mohamed)

Preparación para el cambio: toma de decisión y planificación

Para algunos infractores la mediación fue el lugar adecuado para tomar la decisión de cambiar y prepararse para llevar a cabo dicho cambio. Una vez finalizado el programa de mediación, estaban preparados para cambiar y habían planificado incluso qué deberían de hacer a corto plazo para tener éxito en su nuevo reto. Así pues, el estadio de preparación tiene dos momentos: la toma de decisión y la planificación, en los que es posible identificar a diversos infractores.

En el momento de la toma de decisión es posible identificar a Pau y a Carlos. Este no solamente había tomado la decisión y estaba preparado para cambiar, sino que lo consiguió, según explica en la entrevista. Pau, en cambio, tuvo más dificultades. A pesar de que no reincidió, en la entrevista comenta que sigue teniendo problemas con una de sus necesidades criminógenas más significativas: la adicción al alcohol y al hachís.

En el estadio de la planificación del cambio se encontraban tanto Jonathan como José, y dicen que la decisión ya la habían tomado antes de empezar la mediación, pero que el proceso les sirvió para prepararse para cambiar y hacer frente a las adversidades que el

Revista Española de Investigación Criminológica

Artículo 11, Número 16 (2018)

ttps://doi.org/10.46381/reic.v16i0.189

www.criminologia.net

ISSN: 1696-9219 
cambio podía suponer. Ambos se refieren a estos estadios de forma retrospectiva, ya que en el momento de la entrevista se encuentran en la acción y mantenimiento del cambio.

\section{Acciones para el cambio y mantenimiento del mismo}

Este grupo consiguió llegar a los últimos estadios del cambio propuestos por Prochaska y DiClemente, $(1983,1984)$ : la acción para el cambio y la más desafiante, el mantenimiento del mismo. Este es un grupo compuesto por 3 - Carlos, José y Jonathan- infractores que están clasificados en el grupo de desistentes, en la medida que fueron capaces de mantenerse alejados de la vida antisocial durante los seis meses siguientes al fin de la mediación.

Durante las entrevistas con los infractores de este grupo todos coincidieron en destacar la importancia que había tenido el proceso de mediación para poder cambiar y mantenerse en la consecución de los cambios planteados; así como destacaron otros aspectos positivos en sus vidas que sirvieron de apoyo y motivación para seguir adelante con la vida pro-social.

\section{El proceso de mediación}

En particular, en cuanto al proceso de mediación, destacan que el hecho de haberse encontrado con la víctima les ayudó a tomar conciencia de su comportamiento y les permitió definitivamente decidirse a pasar a la acción y redirigir sus caminos hacia el desistimiento. Estos encuentros les permitieron expresar sus puntos de vista; sentirse escuchados; responder preguntas; reflexionar, lo que encontraron de gran utilidad. Además todos fueron capaces de pedir disculpas y estaban convencidos de que necesitaban hacer frente a sus necesidades criminógenas - adicción a alcohol o drogas; uso de la violencia para resolver conflictos- y poner fin a la actividad antisocial. La mediación fue un refuerzo de ese proceso de cambio.

Jonathan destacó en la entrevista que él ya fue concienciado a la mediación sobre la necesidad de plantearse cambios en su vida, puesto que ya había roto con su pasado. No obstante, la mediación le ayudó a ser reflexivo, especialmente en situaciones en las que antes hubiera respondido violentamente. 


\section{(...) Aja, (.) vale... mmm desde que acabó la mediación ¿qué destacarías, que te} ha pasado?

Pienso más las cosas antes de hacerlas.

Y en tu vida en general, ¿qué cambio ha habido?

No, tranquilidad más que nada, eso de ir más pausado por la vida, no ir tan nervioso ... y y a te digo, reflexionar, pensar las cosas antes de...; analizar la situación en la vida cotidiana antes de alterarse por ejemplo!

¿Crees que te comportas diferente?

Si por asi decirlo! (...)”

(Jonathan)

José reconoce que antes de iniciar la mediación ya se había dado cuenta de que su vida tenía que cambiar de rumbo. Una de los aspectos que destaca haber aprendido durante la mediación fue el de ser reflexivo en situaciones de riesgo, igual que Jonathan.

(...) Y ¿qué te ha aportado a ti la mediación individualmente, a ti mismo, qué cosas destacarías desde que hiciste la mediación?

Pues mira, desde que hice la mediación intento meterme menos en problemas, intento pensar antes de actuar...si, eso! Y si, pensarme las cosas antes de nada! La verdad es que si, me hace pensar bastante: el niño, haz esto bien, no hagas esto mal, no seas tan rencoroso.... a pensar un poco más, porque no me llevaba a ningún lao! Ahora me intereso más por la gente (...) antes simplemente actuaba... ahora me tomo las cosas más...con más suavidad! Para mi ha sido importante esto (la mediación) en la hora de... me ha hecho reflexionar bastante, eso es lo que agradezco, veo que las cosas no las pienso tan a lo bruto y veo que es importante para mi, la verdad! Me ha hecho pensar: frena niño, que no tienes 15 años ahora! Me ha ayudao a...madurar un poquito, por decir algo!

Vale, bueno si tuvieras que resumirme en un titular ¿qué te ha aportado la mediación, qué me dirías?

Mmm.... no sé como decírtelo! Seguridad en mi mismo, el pensar en cómo soy yo; en qué tengo que hacer antes de nada; el conocerme a mí mismo. Me ha ayudao a conocerme, a reflexionar, a pensar en todo, en no tener mala cabeza, me ha ayudao bastante en ser buena persona ¿me explico? (...)

(José)

Factores interpersonales para mantener el cambio

Si bien la mediación fue un elemento significativo en el proceso de cambio de estos chicos y en el mantenimiento del mismo, durante la entrevista hicieron mención a otros factores

Revista Española de Investigación Criminológica

Artículo 11, Número 16 (2018)

ttps://doi.org/10.46381/reic.v16i0.189

www.criminologia.net

ISSN: 1696-9219 
externos que han ayudado y en ocasiones han sido fundamentales para llevar a cabo ese proceso individual de cambio hacia una vida pro-social.

\section{La familia}

Todos- Carlos, Jonathan y José - coinciden en el rol que tuvieron sus familias como elemento motivador y de apoyo en la consecución de este reto y en mantenerlo.

\section{(...) Jóvenes eh! Vale, y ¿cuáles son las personas que has tenido más cercanas durante estos últimos meses $o$ así? \\ Mi madre, mi novia, eh...mi tía, mi hermano...y bueno, algún amigo que otro, mi familia, mis suegros...}

\section{¿Su familia también?}

Sí, por el mero hecho de que están ahí ayudándome, no en economía, sino en consejos! Después ya mi familia ya es tanto económico como emocional.

\section{¿Cómo te ayudan emocionalmente?}

Apoyándome en según qué cosas y en según qué cosas no...que es porque ellos tienen razón! Momento que dices no hijo por esto no, o no hijo por esto sí! No se...si necesitas un abrazo están pa dártelo, si necesitas dos tortas también te lo hacen o sea que (...)

(Jonathan)

Nuevos retos: el trabajo y los estudios

Los participantes en este caso destacan como elementos motivadores externos la realización de cursos de formación o la búsqueda de trabajo.

(...) Bueno y ¿podrías explicar brevemente como ha ido tu vida desde que finalizó la mediación?

Si, he estao estudiando hasta hace nada y después d'acabar esos estudios pues osea, dentro de lo que estoy estudiando hago unas prácticas, y he empezao en una empresa de aires acondicionados, estoy haciendo unas prácticas y me van muy bién. Estoy esperando que me den el coche también y todo bién/ [se refiere al carné de coche]

¿Podrías decir que ha habido un antes y un después?

Si, sin nuevos problemas (...)

(Carlos)

\section{(...) ¿Y tienes trabajo ahora?}

Participante: No, busco. 


\section{Haces algún curso o...}

Sí, estoy haciendo un curso de programación android! Y bueno, se va haciendo!

\section{¿Cómo estás viviendo esto?}

Jodidamente muy jodido! Te ves sin un duro, te ves pues que en tu casa no hay dinero y que no puedes salir... que quieres irte con la novia a algún sitio y no puedes...nose! (...) tienes que pagar cosas y no llega (...) eso de tener que acudir a mis padres por diez euros cuando podría estar ganando mi sueldo y no tener que pedirles nada...pero bueno mira es lo que hay!

(Jonathan)

El abandono del pasado: drogas, barrio y amistades

Todos detectan que para evitar recaídas además de hacer nuevas actividades es necesario abandonar aquellas adicciones, lugares o personas que les mantenían ligados al mundo delictivo.

\section{(...) ¿Has roto la relación con ellos (antiguas amistades)?}

Si he roto la relación, por decirlo así. A lo mejor algún día salgo de fiesta con ellos pero cuando veo para donde van me voy por otro lao y desaparezco.

¿Y en casa con tus padres, que tal?

En casa con mis padres bien, ahora sí. Vamos a cambiar de casa y todo!

Ah! ¿Vais a cambiaros de barrio o de pueblo?

De barrio, bueno se puede decir de pueblo, porque me voy de can1 a can 2, se puede decir de pueblo también (...)".

(Carlos)

\section{(...) Y bueno, un poco más en general ¿cómo es tu relación con las personas más cercanas (familia, amigos)? \\ Sí buena, tenemos nuestras diferencias, pero buena}

\section{¿Son los mismos amigos que los de los dieciséis?}

No, cambié de amigos. O sea, algunos sí, me hablo con ellos y tal, pero no es lo mismo que antes, no me junto con ellos porque no me convienen, los veo, los saludo, hola y adiós y se acabó.

\section{¿Con los que robabas?}

Sí. Estos ya no. Los nuevos son más centrados; No sé, tranquilos, no buscan problemas, no hacen cosas que no convienen...no se! Con los que meramente me junto tienen estudios, trabajan, hacen su vida...algunos están casaos y tienen 22 o 23 años (...)

(Jonathan) 
Perspectivas de futuro

Los tres infractores de este grupo hacen referencia a sus perspectivas de futuro de acuerdo a una vida al margen de la delincuencia, algunos con mayor positivismo que otros. Sin embargo, explican cuál sería su situación límite, la que podría llevarles a meterse en problemas legales de nuevo.

(...) Bueno... ¿cuáles son tus expectativas de futuro?

Yo? Trabajar, tener mi familia, tener mis hijos y vivir mi vida con mi pareja!

¿Qué crees que tienes que hacer para que esto se lleve a cabo?

Yo creo que cambiar los errores que aún sigo cometiendo. No es que cometa los mismos errores que cometía antes, pero quieras que no somos personas y todos fallamos y hay cosas que arreglar siempre!

¿Cómo qué?

Actitudes, y...no se! Actitudes de momentos puntuales, eso de...eh sobresaltos en algún momento o decir no hago esto porque no quiero.

Y de aquí cinco años ¿cómo te ves?

Si sigo en las circunstancias que estoy ahora debajo un puente.

Vale! Y ¿qué crees que podría llevarte a caer de nuevo, a volver a robar a volver a meterte en líos...? Decir, esta situación me llevaría al límite!

Ver que mis hermanos no tienen para comer! Yo eso me... me pierde! A mi me puede faltar de comer, pero a ellos no! (...)

(Jonathan)

\section{(...) ¿Cuál es tu perspectiva en los próximos meses?}

Pues mi perspectiva es conseguir cambiar lo que estoy intentando cambiar

¿Qué es?

El dejar de pelearme con la gente, el dejar de fumar drogas, el tener un trabajo- estoy deseando tenerlo, la verdad!- y si, hacer vida, hacer algo con mi vida! Tener un futuro... lo que al ritmo que llevamos no hacemos nada y tampoco lo... a ver, aquello que no lo piensas mucho y cuando te quieres dar cuenta ya te han pasao siete años y dices, qué has hecho con tu vida! Sí, tengo esa perspectiva de ir cambiando las cosas, hay que arreglarlas, hay que hacer algo!

\section{¿De aquí cinco años como te ves?}

¿De aqui cinco años? Bufff con esta crisis esta que es tan mala? Como me veo, no se... la verdad! Sinceramente no me veo, pero me gustaría verme pos con mi pisito, con mi trabajo, 
con mi dinerito en el bolsillo por si algun dia quiero ir a tomarme esto o... Bueno, madurar! Lo que me quiero referir es a tener la cabeza en su sitio. Pero hay que poner fuerza de voluntad! (...)

(José)

\section{Discusión y conclusiones}

El uso del modelo de los estadios del cambio propuesto por Prochaska y DiClemente (1983) ha permitido examinar el vínculo entre la JR y el desistimiento mediante la descripción detallada del proceso de cambio de los 12 participantes entrevistados seis meses después de la mediación. Los resultados muestran que es posible identificar infractores en todos los estadios del cambio, y que cada proceso es distinto para los participantes.

El objetivo principal relativo comprobar la influencia de la JR en el desistimiento de los participantes en el programa de mediación puede ser confirmado. Con base en los resultados obtenidos, en una amplia mayoría de casos la mediación tuvo un efecto positivo en ayudar a los infractores a iniciar o mantener el proceso de cambio hacia el desistimiento. La mediación fue un espacio en el que los infractores participantes de esta investigación pudieron trabajar y discutir sobre la necesidad de cambiar para evitar reincidir en el futuro. Los elementos relevantes en la teoría del desistimiento se pueden identificar en los procesos de mediación en los que participaron los infractores de los casos analizados; desde la identificación de narrativas de cambio, el trabajo de la autoeficacia, la mejora de habilidades para la comunicación como alternativas hasta la responsabilización y reparación como acciones orientadas al cambio. Sin embargo, hay algunas excepciones, para quienes la mediación no representó un aprendizaje ni influyó en sus vidas, que requieren de un mayor análisis. Sucedió especialmente entre los infractores que se ubican en el estadio de la precontemplación, hecho que los hace poco sensibles al proceso de mediación y, por tanto, parece lógico que no influyera posteriormente.

En esta investigación las narrativas de cambio pudieron detectarse en diversos momentos, por un lado durante las sesiones conjuntas o indirectas; y por el otro, durante la entrevista posterior. Relacionado con la idea de las narrativas de cambio, el cambio en sí 
mismo puede ser explicado a través de los estadios de la teoría del cambio (Prochaska \& DiClemente, 1983, 1984). De hecho, las narrativas pueden ubicarse en los primeros estadios del proceso de cambio, que son los que coinciden con el proceso de mediación. En los estadios más avanzados también se identifican narrativas más de acción y mantenimiento y solamente en algunos infractores.

Concretamente, en cuanto a los estadios de cambio, todos están representados por alguno de los 12 infractores. Hay participantes que se encuentran en los primeros estadios pre-contemplación y contemplación- vinculados a la identificación de la necesidad de cambio, a la toma de decisión de cambiar y a la preparación para hacer frente a tal reto. Es necesario destacar que hay un grupo de infractores que se encuentran en un estadio muy inicial, por no haberse planteado la necesidad de cambiar. Otros, sin embargo, ya durante el proceso pudieron tomar esas decisiones y se encuentran ya en la planificación para pasar a la acción- preparación para el cambio-; mientras que otros manifiestan haber comenzado a encontrar obstáculos que paralizan o dificultan dicho proceso, y que se ubican en la fase de acciones para el cambio. Hay participantes que, no obstante, han ido siendo capaces de superar las adversidades y se encuentran en la constante lucha por mantener la nueva vida estadio de mantenimiento del cambio.

Entre los diversos factores relacionados con la motivación y con el proceso de cambio, la mayoría de los infractores identifican la mediación como un punto de inflexión o elemento de relevancia significativa en ese camino hacia la vida pro-social. Así pues, una idea clave es que la influencia positiva del proceso de cambio puede tener que ver con el estadio de cambio en el que se encuentra la persona en el momento que inicia el proceso de mediación, que además determinará la evolución en los distintos estadios. Esta idea es uno de los resultados obtenidos más relevantes, puesto que permite no solamente hablar de proceso de cambio en general, sino en particular. Para algunos casos, la mediación permite avanzar en un proceso que ya comenzó antes de iniciar la mediación; mientras que para otros la mediación es el primer paso al planteamiento para cambiar. Esto es relevante ya que si se pone énfasis en el trabajo individual con los infractores para generar y promover la necesidad

Revista Española de Investigación Criminológica

Artículo 11, Número 16 (2018)

ttps://doi.org/10.46381/reic.v16i0.189

www.criminologia.net

ISSN: 1696-9219 
de una vida pro-social, los resultados de la mediación pueden ser significativamente más positivos en términos de desistimiento y cambio de rumbo hacia una vida pro-social.

Así mismo, los infractores también destacan factores interpersonales, como el apoyo de sus familias, el mediador $u$ otros aspectos externos como los estudios o el trabajo, como elementos relevantes en el proceso de cambio y, sobre todo, en el mantenimiento de dicho cambio. Los factores interpersonales son importantes para reforzar la identidad convencional y especialmente contribuyen a construir y reforzar la auto-eficacia. Así mismo, es posible detectar que el mantenimiento del cambio después de la JR depende también de los factores contextuales, ya que es posible detectar casos que, si bien la mediación ha sido positiva, la persona ha reincidido.

Por último, cabe destacar que si bien existen casos en los que la influencia del proceso ha sido positiva y los infractores han reincidido, considero que esto debe verse como una recaída en el marco de un proceso, que es el desistimiento y que, por tanto, no puede valorarse negativamente. Al contrario, el proceso de mediación ha contribuido a su avance en los estadios de cambio, con una recaída que generará nuevos aprendizajes en la ejecución de nuevas acciones.

En definitiva, los resultados muestran la complejidad del proceso de cambio y la dificultad que los infractores tienen a la hora de conseguir, pero sobre todo de mantener, este cambio tal y como ha puesto de manifiesto la investigación en el contexto comparado (Bottoms \& Shapland, 2011).

\section{Limitaciones e implicaciones}

En cuanto a las limitaciones asociadas a esta parte cualitativa del estudio es necesario tener en cuenta que los casos analizados ilustran los cambios que los infractores realizan de un estadio a otro, sin embargo, no es posible determinar si dichos cambios han sido significativos.

Además, como implicaciones derivadas de estos resultados, considero que sería necesario trabajar más desde la perspectiva del infractor en clave restaurativa pero

Revista Española de Investigación Criminológica

Artículo 11, Número 16 (2018)

ttps://doi.org/10.46381/reic.v16i0.189

www.criminologia.net

ISSN: 1696-9219 
especialmente de desistimiento, integrando así más elementos relevantes para el desistimiento con el fin de conseguir que la JR forme parte del cambio y permita un proceso real de cambio en los infractores participantes en el mismo. Las necesidades criminógenas deberían ser abordadas desde el principio, durante las sesiones individuales; así como el trabajo sobre la proyección de futuro debería estar presente en todas las fases, y muy especialmente en la sesión conjunta, culminando con su consideración en el documento de acuerdos. Las sesiones individuales son también un espacio trascendental y determinante para conseguir sensibilización, apego y compromiso con el proceso de mediación. De esta forma será posible conseguir una influencia y un impacto positivo para el futuro de los participantes. Finalmente, es necesario garantizar el cumplimiento de los compromisos pactados mediante un buen plan de seguimiento.

\section{Referencias}

Bottoms, A., \& Shapland, J. (2011). Steps towards desistance among male young adult recidivists. In S. Farrall, M. Hough, S. Maruna, \& R. Sparks (Eds.), Escape routes: Contemporary perspectives on life after punishment, (43-80). London: Routledge

Bottoms, A., Shapland, J., Costello, A., Holmes, D., \& Muir, G. (2004). Towards Desistance: Theoretical Underpinnings for an Empirical Study. The Howard Journal, 43 (4), 368-389. DOI: https://doi.org/10.1111/j.1468-2311.2004.00336.x

Braithwaite, J. (2002a). Restorative Justice and Responsive Regulation. Oxford: Oxford University Press.

Braithwaite, J. (2002b). Setting Standards for Restorative Justice. British Journal of Criminology, 42, $563-77$.

Braithwaite, J., \& Strang, H. (2000). Restorative Justice. Philosophy to practice. Burlington: Ashgate

Burnett, R., \& Maruna, S. (2006). The Kindness of prisoners: strengths-based resettlement in theory and in action. Criminology and Criminal Justice, 6 (1), 83-106. DOI: https://doi.org/10.1177/1748895806060668

Christie, N. (1977). Conflicts as Property. British Journal of Criminology, 17 (1), 1-15.

Cid, J., \& Martí, J. (2012). Turning points and returning points: Understanding the role of family ties in the process of desistance. European Journal of Criminology, 9(6), 603-620. DOI: https://doi.org/10.1177/1477370812453102

Daly, K. (2003). Mind the gap: restorative justice in theory and practice. In A. Von Hirsch, J. Roberts, A. Bottoms, K. Roach, \& M.Schiff (Eds.), Restorative Justice and criminal justice: competing or reconcilable paradigms?, (221-236). Oxford: Hart Publishing.

Revista Española de Investigación Criminológica

Artículo 11, Número 16 (2018)

ttps://doi.org/10.46381/reic.v16i0.189

www.criminologia.net

ISSN: 1696-9219 
Dandurand,Y., \& Griffiths, C. (2006). Handbook on Restorative Justice Programmes. New York: United Nations.

Departament de Justícia. (2006). Programa Marc de Mediació i Reparació Penal. Barcelona: Departament de Justícia de la Generalitat de Catalunya.

Dignan, J. (2005). Understanding victims and restorative justice. Maidenhead: Open University Press. McGraw Hill.

Giordano, P., Cernkovich, S., \& Rudolph, J. (2002). Gender, crime, and desistance: Toward a theory of cognitive transformation. American Journal of Sociology, 107(4), 990-1064. DOI: https://doi.org/10.1086/343191

Harris, N., Walgrave, L., \& Braithwaite, J. (2004). Emotional dynamics in restorative conferences. Theoretical Criminology, 8, 191-210. DOI: https://doi.org/10.1177/1362480604042243

Harris, N. (2006). Reintegrative Shaming, shame and criminal justice. Journal of Social Issues, 62, 327-346. DOI: https://doi.org/10.1111/j.1540-4560.2006.00453.x

Hoyle, C., Fonseca, F. (2016). Looking back to the future: threats to the success of restorative justice in the United Kingdom. Victims \& Offenders, 11:30-49. DOI: https://doi.org/10.1080/15564886.2015.1095830

Johnstone, G., \& Van Ness, D. (2007a). The meaning of restorative justice. In G. Johnstone, \& D. Van Ness. Handbook of Restorative Justice (1-23). Cullompton, Devon: Willan Publishing.

Johnstone, G., \& Van Ness, D. (2007b). Evaluation of restorative justice. Introduction. In G. Johnstone, \& D. Van Ness. Handbook of Restorative Justice (395-397). Cullompton, Devon: Willan Publishing.

Karp, D. (2001). Harm and repair: Observing restorative justice in Vermont. Justice Quarterly, 18 (4), 727-757. DOI: https://doi.org/10.1080/07418820100095081

Marshall, T. (1999). Restorative Justice: An Overview. Home Office Occasional Paper. London: Home Office.

Maruna, S. (2001). Making Good. How ex-Convicts Reform and Rebuild their Lives. Washington: American Psychological Association.

Maruna, S. (2014). Plenary session on desistance. Invited plenary presentation to the 8 th Conference of the European Forum for Restorative Justice. Belfast, 11-14 June.

Mulvey, E. P., Steinberg, L., Fagan, J., Cauffman, E., Piquero, A. R., \& Chassin, L. (2004). Theory and Research on Desistance from Antisocial Activity among Serious Adolescent Offenders. Youth Violence and Juvenile Justice, 2 (3), 213-236. DOI: http://dx.doi.org/10.1177/1541204004265864

Presser, L., \& Van Voorhis, P. (2002). Values and evaluation: Assessing Processes and Outcomes of Restorative Justice programmes. Crime and delinquency, 48, 162-188. DOI: https://doi.org/10.1177/0011128702048001007

Prochaska, J; \& DiClemente, C. (1983). Stages and processes of self-change of smoking: Toward an integrative model of change. Journal of Consulting and Critical Psychology, 51, 390-395.

Prochaska, J; \& DiClemente, C. (1984) The transtheoretical approach: Crossing traditional boundaries of therapy. Malabar, FL: Krieger.

Revista Española de Investigación Criminológica

Artículo 11, Número 16 (2018)

ttps://doi.org/10.46381/reic.v16i0.189

www.criminologia.net

ISSN: 1696-9219 
Roberts, M. (2010). Evaluating evaluation: an investigation into the purpose and practice of evaluation in restorative justice based programmes. Thesis. Canada: Simon Frase University.

Robinson, G., \& Shapland, J. (2008). Reducing recidivism. A task for Restorative Justice?. British journal of Criminology, 48, 337-358. DOI:https://doi.org/10.1093/bjc/azn002

Rossner, M. (2011). Emotions and interaction ritual. A micro analysis of restorative Justice. British Journal of Criminology, 51, 95-119. DOI:https://doi.org/10.1093/bjc/azq075

Scheff,T., \& Retzinger, S. (1991). Emotions and violence: Shame and Rage in Destructive Conflicts. Lexington, MA: Lexington Books.

Shapland, J. (2013). Desistance from crime and the potential role of restorative justice. Paper given at the conference 'What is criminology?', to celebrate the work of Sir Anthony Bottoms, Cambridge, 30 November 2007.

Shapland, J., Atkinson, A., Atkinson, H., Chapman, B., Dignan, J., Howes, M., Johnstone, J., Robinson, G. \& Sorsby, A. (2007). Restorative Justice: the views of victims and offenders. The Third Report from the Evaluation of Three Schemes. London: Ministry of Justice.

Shapland, J., Robinson, G. \& Sorsby, A. (2011). Restorative Justice in Practice. Evaluating what works for victims and offenders. London: Routledge.

Strelan, P., Di Fiore, C., Van Prooijent, J. (2017). The empowering effect of punishment on forgiveness. European Journal of Social Psichology, 47 (472-487). DOI:https://doi.org/10.1002/ejsp.2254

Van Stokkom, B. (2002). Moral emotions in restorative justice conferences: Managing shame, designing empathy. Theoretical Criminology, $6 \quad$ (3), 339-360. DOI:https://doi.org/10.1177/136248060200600306

Varona, G. (2008). Evaluación de la actividad del servicio de mediación penal de Barakaldo (juliodiciembre de 2007). Donostia- San Sebastian.

Zehr, H. (1990). Changing lenses: A new focus for crime and justice. Scottdale, PA: Herald Press.

Walgrave, L. (2007). Restorative Justice, Self-interest and Responsible Citizenship. Cullompton, Devon UK: Willan Publishing.

\section{Agradecimientos}

Quiero agradecer a la SEIC por haberme concedido el premio a joven investigadora y así darme la oportunidad de publicar este artículo. Así mismo, agradezco la ayuda brindada por el Prof. José Cid en las versiones más recientes de este artículo, por sus aportaciones a la mejora del texto.

Anna Meléndez es doctora en derecho por la Universidad Autónoma de Barcelona (UAB). Es investigadora post-doctoral y trabaja como profesora impartiendo clases en el grado de criminología de la UAB y es sub-coordinadora del mismo. Sus líneas de investigación son la justicia restaurativa, las alternativas a la prisión y el desistimiento.

https://orcid.org/0000-0002-4654-4957

\section{Revista Española de Investigación Criminológica}

Artículo 11, Número 16 (2018)

ttps://doi.org/10.46381/reic.v16i0.189

www.criminologia.net

ISSN: 1696-9219 